\title{
Albanon
}

Revistë kulturore

\section{Regjistri i Trikallës i vitit 1455 dhe të dhëna për historinë shqiptare dhe shqiptarët}

Ermal Nurja

Në vitin 1455 sulltan Mehmeti i II, duhet të ketë urdhëruar një regjistrim të timareve në gjithë Perandorinë Osmane. Nuk ka mbërritur asnjë shkresë e kohës që vërteton urdhrin e sulltanit, por fakti që një numër i madh regjistrash që kanë mbërritur në ditët e sotme mbajnë këtë datë, pra vitin 1455, vërteton që sekretaria e timareve të ketë hartuar një regjistrim të tillë. Regjistra të tillë kanë mbërritur për Rumelinë edhe për Anadollin.

Regjistrimi në vitin 1455 ka edhe një logjikë të re. Tashmë në duart e osmanëve kishte rënë Kostandinopoja, për marrjen e së cilës ishin mobilizuar të gjitha forcat ushtarake; tashmë perandoria do të kishte një kryeqytet të ri, prandaj regjistrimi do të hidhte dritë mbi statistikën e fundit të perandorisë.

Një nga regjistrat e vitit 1455 është edhe regjistri i sanxhakut të Trikallës (Tırhala sancağı). Regjistri ruhet në Arkivin Osman në Stamboll (BOA), në fondin MAD nën numrin 10.

Regjistri është botuar në dy vëllime në vitin 2001, nga Instituti i Historisë Turke në Ankara. Ka dy autorë Melek Delilbashë (Delilbaşı) dhe Muzafer Arëkan (Arıkan). Në vëllimin e parë autorët kanë paraqitur të dhënat historike që përmban regjistri në lidhje me forcat ushtarake: aplikimin e sistemit të timareve, të dhënat mbi financën osmane (taksat), prodhimet bujqësore që përfshihen në sistemin e taksave.

Jo vetëm kaq, por ata kanë transliteruar në këtë vëllim të dhënat e timareve, timarlinjve, listat me emrat e popullsisë myslimane, të dhënat financiare dhe ushtarake që kishte çdo timar dhe të dhënat e popullsisë.

Regjistri në fjalë është i tipit $i$ detajuar (mufasal), që do të thotë se përveç të dhënave të përmendura më sipër, përmban edhe listat me emrat e kryefamiljarëve të vatrave (hane), beqarëve dhe vatrave që kishin kryefamiljare të veja (bive). 
Këto lista nuk janë të transliteruara në vëllimin e parë. I gjithë regjistri është botuar në origjinalin osmanisht në vëllimin e dytë.

Gjatë regjistrimit të familjarëve, beqarëve dhe të vejave, regjistruesi osman lejon personin të regjistrohej në atë mënyrë që ai identifikohej. Nuk është e qartë se në çfarë mase ky lloj regjistrimi ndikohej nga regjistrimet e mundshme paraosmane të periudhës bizantine.

$\mathrm{Në} \mathrm{regjistër} \mathrm{personat} \mathrm{regjistrohen} \mathrm{mes} \mathrm{të} \mathrm{tjerash} \mathrm{edhe} \mathrm{sipas} \mathrm{etnisë.} \mathrm{Kështu}$ personat janë regjistruar edhe si sllavë (islavis, isklavis), grekë (rum, rumiyus, igrekus), vllehë (evlahos, evlasis) dhe shqiptarë (arnavud, arnavidis).

Forcat osmane në Thesali komandoheshin nga familja e Evrenozëve, një familje shqiptare nga Dibra që kishin bërë karrierë në ushtrinë osmane.

Forcat osmane të Trikallës goditën në shpartallim forcat spanjolle që kishin rrethuar Beratin në bashkëpunim me Skënderbeun.

Sanxhaku i Trikallës në vitin 1455 përbëhej nga tri kaza që ishin: Trikalla, Feneri dhe Agrafa.

Qytetet e regjistruara janë: Trikalla, Larisa (Jenishehir), Feneri dhe Agrafa. Kala e regjistruar është vetëm ajo e Damsit. Ekzistonte edhe nahija e Radovizit nën varësinë e kazasë së Fenerit dhe treva (il) që duhet të kenë ekzistuar si njësi që në kohën para vendosjes së sundimit osman, Kravar ili dhe Mjekra ili. Kjo e dyta nga autorët e botimit është transliteruar në formën Mikira, që e konsideroj të gabuar.

Popullsia e sanxhakut të Trikallës është si më poshtë':

\begin{tabular}{|l|r|r|r|r|r|r|}
\hline & \multicolumn{3}{|c|}{ Mysliman } & \multicolumn{3}{c|}{ Jomysliman } \\
\hline & Vatra & Të veja & Beqar & Vatra & Të veja & Beqar \\
\hline Trikalla qytet & 251 & 9 & 0 & 212 & 73 & 0 \\
\hline Larisa qytet & 355 & 12 & 0 & 66 & 17 & 0 \\
\hline Fener qytet & 146 & 5 & 0 & 321 & 96 & 0 \\
\hline Agrafa qytet & 0 & 0 & 0 & 327 & 60 & 5 \\
\hline Trikalla kaza & 992 & 50 & 0 & 1300 & 1349 & 1157 \\
\hline Fener kaza & 790 & 46 & 0 & 5261 & 700 & 394 \\
\hline Agrafa kaza & 133 & 19 & 0 & 3801 & 166 & 541 \\
\hline Total & $\mathbf{2} \mathbf{6 6 7}$ & $\mathbf{1 4 1}$ & $\mathbf{0}$ & $\mathbf{1 1 ' 2 8 8}$ & $\mathbf{2}^{\prime} \mathbf{4 6 1}$ & $\mathbf{2}^{\prime} \mathbf{0 9 7}$ \\
\hline
\end{tabular}

1 Hicri 859 Tarihli Suret-i Defter-i Sancak Tırhala, vol. 1, fq. XXVI-XXXII. 


\section{Albanon}

Revistë kulturore

Forcat ushtarake osmane në sanxhakun e Trikallës janë si më poshtë2:

\begin{tabular}{|l|c|c|c|c|c|}
\hline & Subash & Timarlinj & Mbrojtës kalash & Xhebelu & Ogllan \\
\hline Trikalla kaza & 1 & 88 & 24 & & \\
\hline Fener kaza & 1 & 63 & 10 & & \\
\hline Agrafa kaza & 1 & 7 & 0 & & \\
\hline Total & 3 & 158 & 34 & 340 & 68 \\
\hline
\end{tabular}

Në kazanë e Trikallës nga 112 timare, 89 u takonin timarlinjve myslimanë, ndërsa 20 timare u takonin timarlinjve të krishterë. Në kazanë e Fenerit nga 73 timare, 61 ishin timarlinj myslimanë, ndërsa 12 të krishterë. Në kazanë e Agrafas nga 7 timare, 6 ishin timarlinj myslimanë, 1 ishte i krishterë. Në total në sanxhakun e Trikallës nga 192 timare në total, 156 janë myslimanë, 33 të krishterë dhe 3 janë të përzier (mysliman dhe jomysliman). Një timar ka statusin vakant (mevkuf).

Në listën e timarlinjve të sanxhakut të Trikallës, ka të dhëna historike si për timarliun Mahmud, serhani është dizdari i kalasë së Beratit në Shqipëri (76a), ndërsa tek timari i Ibrahimit birit të Mukbilit, Jusufit dhe Hamzait bijve të Ahadit. Ibrahimi është turk i ardhur nga Anadolli, ndërsa dy të tjerët janë minorenë. Baballarët e tyre kanë rënë në luftimet e kalasë së Koxhaxhikut. (79b).

Një pjesë e timarlinjve të sanxhakut të Trikallës, veçanërisht ata të krishterë, duket qartë se janë shqiptarë, siç janë Muriqi dhe Gjini (101b), Miho biri i Klaznos dhe Aranidi biri i Vradinos (130a), Dimo, Jorgji dhe Kirjo Duka dhe Mazaraku bijtë e Dimos (143b), Lalsa dhe i vëllai Kosta (145b), Gjini vëllai i Stefanit dhe Jani i biri i Stefanit (179b), Gazi dhe Nikolla bijtë e Bardhit (183b), Mazaraku vëllai i Janit dhe Gjini (187a), Ibrahimi dhe Jusufi bijtë e Isa beut biri i Pavlo Kurtiqit (212a), Gllava, Domeniku, Mazaraku bijtë e Mjekrës (232a), Komenosi dhe vëllai tij Muzaka (233b), Mustafa dhe Petro bijtë e Mjekrës (234b), Pavl biri Mjekrës (236b), Moisiu, Karaxha, Petri madh dhe Kafrli bijtë e Kravarit (239b), Teodor biri i Muzakut dhe Brajko biri i Teodorit (317a), Gjoni dhe Seraqini (318a), Gjon Mavromati (341a), Gjin Mazarak dhe i vëllai Teodori (341b), Sylejmani dhe i vëllai İljasi, janë shqiptarë (347a), Mjekra, Kokara dhe Likureshi (348a), Gjin Likureshi dhe Llazar Likureshi, janë shqiptarë (348b), Kollza dhe Aranidi bijtë e Arestit, të parët e tyre kanë qenë kethydatë (ndihmës komandant) e Trikallës (354a), Bardhi dhe Teodori bijtë e Gjonit (450a),

2 Hicri 859 Tarihli Suret-i Defter-i Sancak Tırhala, vol. 1, fq. XLI. 
Gjurmë të shqiptarëve gjenden edhe te truallishta e Kozjakut, që quhet ndryshe edhe bjeshka e shqiptarëve (190b),

Gjatë studimit të regjistrit të Trikallës, kemi të dhëna edhe për popullsinë shqiptare. Të dhënat ndahen në tri grupe që janë: të dhëna për lagje dhe fshatra totale me banorë shqiptarë, të dhëna për shqiptarë në lagje dhe fshatra dhe të dhëna për shqiptarë që kuptohen nga emrat e tyre të fiseve (muzak, mazarak etj.). Bazuar në këtë klasifikim janë përgatitur edhe listat e mëposhtme:

\section{a) Në qytetin e Trikallës gjendet një lagje që quhet lagjja e shqiptarëve (mahalle arnavudan) ku banonin 27 vatra dhe beqarë si dhe 4 të veja (7b).}

Ka edhe fshatra të tëra me banorë shqiptarë që quhen edhe fshatra shqiptare siç janë fshati Çuka me 47 vatra, 5 të veja dhe 10 beqarë (33b), Arnavudan me 23 vatra, 5 të veja (157a), Vila me 15 vatra dhe 3 të veja (këta shqiptarë kanë qenë të falur nga taksat nga Turhan beu, ndërsa tani janë regjistruar si raja) (159b), Toshkës me 16 vatra, 2 të veja (268a), Muzaka me 66 vatra dhe 3 të veja (318a), Kondostavlo me 21 vatra, 3 të veja (356b), Koçohir me 11 vatra dhe 2 të veja (362b).

\section{b) Në lagjen Birgi të qytetit të Trikallës janë regjistruar Dogan arnavudi (3b) dhe djali i tij Jusufi (4a).}

Trikalla qyteti, lagjja 3: Dimas arvanidis, Mihos arvanidis, Gjurkas arvanidis; lagjja 5: e veja e Nikolla arvanidis (5a-5b).

Fshati Bihonishta: e veja Teodora arvanid, Milkos arvanid (13b),

Fshati Perabrishtani varet nga Feneri: Janis arvanid (15b),

Fshati Kalojevrohori: Jorgji arvanid (16a),

Fshati Vinota: Miros arvanid (17b),

Fshati Milova: Nikolas arvanid (19a), İstefanos arvanid (20a),

Fshati Seniç: Gjinis arvanidis (20b),

Fshati Klapodi: Martinis arvanidis (27a-b),

Fshati Destekane: Gjinis arbanidis (28a),

Fshati Konsko: Janis arvanidis, Andreyas arvanid, Mihalis arvanid (30a)

Bratos arvanid, İstefanos arvanidis, Likas arvanid (30b),

Fshati Komat: Nikolas arvanidis, Didyas arvanidis (31b),

Fshati Kirjonero: Demetris arvanidis (32b), 


\section{Albanon}

Revistë kulturore

Fshati İflamuri: Nikolas arvanid, Mihalis arvanid (35a),

Fshati Mavril: İstefanos arvanid (36b),

Fshati Vaniya: Dimos arvanidis (41a), Minkulas arvanidis (42a),

Fshati Lobras: Janis arvanidis, Mihalis arvanidis, Jorgji arvanidis, Rados arvanidis (43a),

Fshati Miçova / Miçoya: Nikolas arvanidis, Jorgji arvanidis (45b), Jorgji arvanid (46a),

Fshati Tiravatitena varet nga Meçova: Gjinis arvanid (48b),

Fshati Mila varet nga Meçova: Janis arvanos, Nikolas arvanos, Janis arvanos, Nikolas arvanidis, e veja Arvanida (50a) Jorgji arvanos, Likas arvanidis (50b), Kiralis arvanidis (51a), İstefanos arvanidis, Tevodoros arvanidis, (52a),

Qyteti Larisa: Nikolas arvanidis (57b),

Fshati Halapos: Mihalis arvanid, Jorgji arvanid, Nikolas arvanid (63b), Kostas arvanidis, Likas arvanid (64a),

Fshati İsparmo: Jorgji arvanid (69a),

Fshati Zarko: Tevodoros arvanidis (72a),

Fshati Seka: Likas arvanid, Kondos biri i arvanid (73b), Dimos arvanidis, Kostas arvanid (74a),

Fshati Tirena: Mekşas arvanidis, İstefanos arvanidis (76a),

Fshati Papadhapulos: Buykos arvanidis, Kozmas arvanidis, Gjonis vëllai i Kozma arvanid, Nikolas arvanidis, Tevodoros arvanidis, Andreyas arvanidis, Gjinis arvanidis, diğer Andreas arvanidis (90b),

Fshati Pirator: Jorgji arvanidis (91b),

Fshati Zhuljani: Kostas arvanidis (92b),

Fshati Qirjavershi ndryshe edhe Malathera: Dimos arvanidis (95a),

Fshati Ayo Demetri: Jorgji arvanidis, Janis arvanidis, Janis arvanidis tjetër (99a), Jorgji arvanidis (99b),

Fshati Kamira varet nga Fenar: Tevodoros arvanidis (100b),

Fshati Zaravil: Lazaris arvanidis (103b), İstefanos arvanidis (104a),

Fshati Velçiç varet nga Allasonja: Janis arvanidis (106b),

Fshati Lavni / Eroni: Likas arvanidis (113a),

Fshati Pavrish: Gjonis arvanidis (113b),

Fshati Paliya Obroshani: Martinis arvanid, Ostoyos arvanidis (119a),

Fshati İstagos ndryshe edhe Kalbak Kaja: Andreas arvanidis, İrbiçis arvanidis, Demetris arvanidis (120b),

Fshati Raksha: Mazas arvanidis (142b),

Fshati Baltenos: Janis arvanidis, Kostas arvanidis, Jorgji arvanidis, Kostas arvanidis (147a), 
Fshati Doksiseta: Jorgji arvanidis (155b),

Fshati Zavlinda: Dukas arvanidis (161b),

Fshati Seroveta: Janis arvanidis (162b),

Fshati Nomi: Gjinis arvanid, Mihalis arvanid (165a),

Fshati Kordiq varet nga Fenar: İstanish arvanidis (167b),

Fshati Lezizhta: Mihalis arvanidis (168b),

Fshati Zirç: Gjinis arvanid (173b),

Fshati Vilvizda: Tevodoros arvanidis (197a),

Fshati Lebanova: Nikolas arvanid (203b),

Fshati Kastanya: Ratinos arvanid (205a),

Fshati Radani: Mihos arvanidis (205b),

Fshati Kurmara: Dimos arvanid, Mekshas arvanid, Jorgji arvanid, Kondos arvanid (206b),

Fshati Tihova: Gjonis arvanid, Andreas arvanid, Lulas arvanid, İvretos arvanid (207a),

Fshati palya Nishtani: Dimos arvanid, İstefanos arvanidis (208b),

Fshati Grazhdani: Ostojos arvanid (209b),

Fshati Aya: Nikolas arvanidis, Garos arvanidis, İstamadis arvanidis, Nenadas arvanidis (218a),

Fshati Thenatos: Mihalis arvanidis (220a),

Fshati Çınarlu ndryshe edhe Sakolova: Eliyas arvanidis, Jorgji arvanidis (220b),

Fshati Karpinish: Jorgji arvanidis (224a), Kostas biri i Jorgji arvanidis (224b),

Fshati Kirgjegj madh: Menkshas arvanidis (235a),

Fshati Rahova: Nikolas arvanidis (247b),

Fshati Vil viçeta: Jorgii arvanidis (251b),

Fshati Tiflosin: Barkthimis arvanid (255b),

Fshati Kuzthini Valkanos: Dimitris arbanjodis (256a),

Fshati Dhomokos: Kostas arbanasis (261a), Nikolas arbanasis (262a),

Fshati Karis: Mekshas arvanid (263b),

Fshati Dhorovi: Gjonis arvanid, Dimitris arvanidis,

Fshati Qirazoj: Dimitris arvanid, Janis arvanid, Jorgji arvanid (286b),

Fshati Mashkullori: Eliyas arvanid (291b),

Fshati Ziniç varet nga Radoviz: Andreyas arvanid (297b),

Fshati Petriç: Gjinis arvanid (306b),

Fshati Palama: Gjonis arvanidis, Jorgji arvanidis (314b),

Fshati Franku: Manolis biri i Jani arvanid (317a), 


\section{Albanon}

Revistë kulturore

Fshati Kolnaris: Dimitris arvanid (325a),

Fshati Komazas: Gjinis arvanid (332a),

Fshati Molesko: Biros arvanid (344a),

Fshati Korbura: Dimitris arvanidis (345a),

Fshati Biçari: Kostas arvanidis (346a),

Fshati Kadosh ndryshe edhe Pirashina: Likas arvanidis (349b),

Fshati Kolidha: Janis arvanid (354b),

Fshati Markos: Mihalis arvanidis (355a) Domenikas arvanidis (355b),

Fshati Doros: Janis arvanidis, Tevodoros arvanidis, Dimitris arvanidis, Jorgji arvanid, Janis Mazaraki (359b), Mihalis arvanid (360a),

Fshati Katos: Jorgji arvanidis (378a),

Fshati Kadhqeli: Senis arvanidis (382b),

Fshati Puljana: Gjonis arvanidis, Dimitris arvanidis, Tevodoros arvanidis (395a),

Fshati Hirsova: Tevodoros arvanidis, Janis arvanid, Gjonid arvanid (425a),

Fshati Karvono Bilishi: Gjonis arvanid (428b), Jorgji arvanid (429a),

Fshati Bizhila: Jorgji arvanidis, Janis arvanid (435a) Jorgji arvanid (435b), Fshati Kalençi: Mihalis arvanidis, Lakas arvanidis (440a),

Fshati Magula: e veja arvanidçe, Janis biri i arvanidçe, Senis biri i arvanidçe (441a),

Fshati Pizhujani: Jorgji arvanid (442a), Dimitris arvanid (442b),

Fshati Shoprila / Thevo Pirila: Janis arvanid (451b).

\section{c) Fshati Seniç: Domenikas Mazaraki (2ob)}

Fshati İzmiçi: Nikolas Mazaraki, Tevodoros Mazaraki (25b, 26a),

Fshati Kirçota: Kostas Mazaraki (26a),

Fshati Klapodi: Kostas Malakas, Gjonis Aranidis, Nikos Mirashis, Dimos Mirash (27a-b),

Fshati Konsko: Bogdanos Muzhaki, Dimitris Muzakis (30a),

Fshati Kata Kladis: Gjinis Muzakis (30b),

Fshati Lobras: Janis Muzakis (42b),

Fshati Velimishta: Dimos Mazaraki (43b),

Fshati kato Kitona varet nga Meçova: Nikolas Martinis, Kostas Martinis (47b),

Fshati Tiravatitena varet nga Meçova: Tevodoros İspatas, papas Martinis (48b),

Fshati Mila varet nga Meçova: Janis Martinis (52a), 
Fshati Koçikova: Janis Tanush, Mihalis Tanush, Manolis Tanush (87b), Tanush biri i Tanush (89a),

Fshati Zhuljani: Mihalis Martinis, İstamadis Martinis, Janis Martinis, Demetris Martinis (92b),

Fshati Papadhopulos: İstefanos Buykos (90b),

Fshati Kokalas: Lazaris İspatas, Gjonis İspatas (100a),

Fshati Velçiç varet nga Allasonja: Demetris Mazaraki, Mihalis Mazaraki, (106b) Manolis Mazaraki, Jorgji Mazaraki (107a),

Fshati Petra: Manolis Malakasis, Jorgji Malakasis (107b), Qiryakos Malakas, Dimos Malakas (108a),

Fshati Virneçja: Garos Martinis (117b),

Fshati İspata: Janis İspatas, Tevodoros İspatas, Gjonis İspatas, Janis İspatas, İstamadis İspatas (118a),

Fshati Samosal: İstamadis Martinis (129a),

Fshati Baltenos: Kostas Radoslav, Thetoqis Radoslav, Aleksi Radoslav, İstamadis Radoslav (147a),

Fshati Asprukilisa: Janis Radoslav (191a),

Fshati Kolondra: Jorgji biri i Matarangos, Radoslavos Matarangos, Janis Matarangos, Tevodoros Matarangos (202b),

Fshati Aya: Dabzhivos Muzhaki, Janis Dabzhivos, Dimos Dabzhivos (218a),

Fshati Thenatos: Mekshas Metarangas (220a),

Fshati Çınarlu ose ndryshe Sakolova: Gjinis Matarangas (220b),

Fshati İnblaki: Jorgji Mazarakis (222a),

Fshati Karpinish: Dimitris Muzaki, Mekşas Malakas, Jorgji Muzaki (225a) Gjinis Malakas (225b),

Fshati Rahova: Gjonis Malakas is (239b),

Fshati Birbijani: Jorgji Bardhas, Nikolas Materangas, Janis Matarangas (245a),

Fshati Simo: Kostas Muzakis, Malakasis Muzakis, Kostas Muzakis (251a), Fshati Filipa: Likas Malakasis (252b),

Fshati Karis: Nikolas gjirokastriti (ergirikastris) (263b),

Fshati Mashkulori: Gjonis Radoslav (291b),

Fshati Gjin Lalthi: Gjinis Muzaki (306a),

Fshati Velçina: Dimitris Martinis, Jorgji Martinis, Tevodoros Martinis (312b), Jorgji Martinis, Nikolas Martinis (313a),

Fshati Kolnaris: Qiryakos Martinis (325a),

Fshati Qilalthi: Mihalis Mazaraki, Qilalthis Malakasis (331b), 


\section{Albanon}

Revistë kulturore

Fshati Komazas: Gjinis Mazaraki, Nikolas Mazaraki, Jorgji Mazaraki (332a),

Fshati Mavromat: Gjonis Martinis (340a),

Fshati Malakas: Tevodoros Muzaki, Dimos Muzakis, Gjinis Muzakis (341b),

Fshati Peçi: Dimitris vëllai i Gjinis, Gjonis Muzaki, Gjinis Muzaki, Gjinis Mazaraki, Jorgji Mazaraki, Kostas Mazaraqi, Gjinis biri i Gjonis, Nikolas Likas, İvretos vëllai i Martinos, Ashtinis Muzaki, Ashtinis vëllai i Gjinis (347b),

Fshati Malaş: Tevodoros Gjinis (349a),

Fshati Kolça: Jorgji Radoslavis, Mihalis Martinis, Jorgji Martinis (351a), Fshati Mezdan / Nezdan, vakëfi i Burak beut të ndjerë, Mihalis Muzhaki (371a),

Fshati Pirti: Mihalis Malakas, Kostas Malakas, Nikolas Malakas (376a), Janis Martinis (376b) Manolis Malakas (376b),

Fshati Seraqeno: Janis Muzakis (382a),

Fshati Vonista: Jorgji Radomiro, Kostas Radomiro (383a),

Fshati Kotlani: Janis Martinos, Nikolas Martinos, Tevodoros Martinos, Tevodoros Martinos tjetër, Dimitris Martinos, Aleksi Martinos, Manolis Martinos (416a),

Fshati Viranyana: Manolis Radoslav (417a) Nikolas Muriqis, Manolis Muriqis (417b), Janis Radoslav (418a),

Fshati Hirsova: Janis Malakas, Nikolas Malakas, Dimitris Malakas (426b),

Fshati Keleçu: Dimitris İstanislav, Mihalis Radomiro (445b),

Fshati Gjare varet nga Agrafa: Janis Novakos, Manolis Novakos (458a). 